\title{
Adrenopause - does it really exist?
}

\author{
Lucyna Papierska
}

Department of Endocrinology, Centre of Postgraduate Medical Education, Bielański Hospital, Warsaw, Poland

\begin{abstract}
In ageing human adrenal glands there occur some morphological changes which result in alterations of their cortex endocrine function. Glucocorticoid-excreting cells in the zona glomerulosa live longer than androgen-producing cells in the zona reticularis, which undergo significant apoptosis. Therefore, in elderly humans cortisol levels are normal (significantly higher than at young age), while adrenal androgen concentrations decline with ageing. Function of the zona glomerulosa is affected by the adrenal status, circulatory system condition, efficiency of the kidneys and liver and medication. An important problem of ageing is the rising incidence of non-secreting, incidentally detected, benign adrenal tumors, called incidentalomas. They necessitate clear-sighted radiological and hormonal diagnosis.
\end{abstract}

Key words: adrenal cortex, adrenopause, glucocorticoids, DHEA, adrenal incidentaloma.

It is a well-known fact that the ageing processes affect, among others, the endocrine system. The phenomenon of menopause in about-50-year-old women corresponds with sudden cessation of cyclical estrogen secretion by the ovaries. In men, often already at the age of 40 , the testosterone secretion from the testicles decreases. It is also known that with age the frequency and amplitude of pituitary pulses of growth hormone decrease, and that the evening melatonin pulse from the pineal gland declines. The term "adrenopause" usually denotes the decrease of blood concentration of adrenal androgens in ageing persons. However, these are not the only changes in the function of the adrenal cortex in the process of ageing of the organism.

The adrenal cortex consists of three layers: the glomerular zone - external, secreting mineralocorticoids (aldosterone); fascicular - located deeper and secreting glucocorticoids (mainly cortisol); and finally, the deepest reticular zone, secreting androgens (dehydroepiandrosterone [DHEA] and its sulfate [DHEAS]). The stimulating action of the pituitary corticotropin (adrenocorticotropic hormone $[\mathrm{ACTH}]$ ) exerts a response mainly in the fascicular zone and, although to a lesser degree, also in the reticular one. The glomerular layer, independent of ACTH, is the last element of the renin-angiotensin-aldosterone system. The processes occurring with age in the adrenal glands affect their whole cortex and may influence the secretion of all of the three groups of its hormones [1].

\section{Glucocorticoids}

The secretion of most of the hormones in humans decreases as the process of ageing advances. That rule does not apply, however, in the case of glucocorticoids. It has been found that the concentrations of cortisol increase with age. It may refer to the morning peak of cortisolemia, as well as to the cortisol concentration in evening hours and to the overall sum of hormone secreted daily [2-5]. In some papers, a larger increase of cortisol concentration in serum under stress in elderly persons than in young and healthy is described. Those phenomena are related, inter alia, to the decrease of sensitivity of the hypothalamus and pituitary gland to inhibition by cortisol [6]. No differences in response to low $(1 \mu \mathrm{g})$ doses of (1-24)ACTH have been found; however, after $250 \mu \mathrm{g}$, which is a dosage usually used in the test of adrenal cortex simulation, in healthy elderly patients a significantly larger increase of cortisolemia has been found [7]. On the other hand, elderly patients in septic shock have the output cortisol concentrations admittedly higher, but a smaller increase after stimulation with ACTH is found; therefore, in those patients socalled functional adrenal insufficiency is found significantly more frequently [8, 9]. In patients with depression at elderly age particularly high cortisol concentrations are observed. Those can have a negative impact on the central nervous system: they accelerate the processes 
of degeneration in the hippocampus, deteriorate recent memory, and aggravate sleep problems [10, 11].

Even a slightly decreased concentration of cortisol in elderly patients should not therefore be ignored. A senile person should physiologically have their cortisolemia at the same levels as a young person, or perhaps even somewhat higher. The first and only, and a very characteristic, symptom of the disease may be orthostatic hypotony, mistaken for an effect of the age-related processes (arteriosclerosis, the decrease of sensitivity in baroreceptors), or a result of medications (hypotensive drugs, dopamine agonists) [12]. Moreover, the adrenal insufficiency may last for years in the form of chronic fatigue syndrome, muscular and articular pains, or the loss of appetite - ailments common in elderly persons. Evaluating the additional examinations, one should pay attention to the concentrations of electrolytes in serum. Hyponatremia in every patient, including in advanced age, requires considering, among other things, adrenal insufficiency in the differential diagnosis [13]. During decreased adrenal reserve, intensification of any senile disease may lead to adrenal crisis. Early diagnosis is therefore very important [14].

\section{Adrenal androgens}

The most characteristic feature of "ageing" of the adrenal cortex is decreased secretion of adrenal androgens - DHEA and DHEAS. The function of these hormones isn't fully discovered yet, but it is known that in considerable amounts they are produced only in adrenal glands of primates. Their concentration in serum, being high in the fetus (DHEA is a substrate for placental production of the estrogens), falls to trace amounts in the first few weeks after birth, and increases only at the beginning of puberty (adrenarche anticipates menarche). Starting already at the age of 40 , a gradual decrease of DHEA secretion is observed $[15,16]$. Those changes occur in both sexes, but in women the concentrations of dehydroepiandrosterone and its sulfate are lower. DHEA influences the receptors of the central nervous system (it is a GABA antagonist) and estrogen receptors (antagonist-agonist action), and has its "own" receptor in lymphocytes. It is also, and according to some researchers first and foremost, a precursor hormone. In elderly men, $50 \%$ of the sex hormones comes from circumferential conversion of DHEA to testosterone. Adrenal androgens are a source of almost $100 \%$ of active estrogens in women after menopause [17].

The physiological importance of DHEA in primates is not fully known. However, on the basis of cross-sectional epidemiological research and observational studies, possible effects of its insufficiency have been described. In men and women, DHEA deficiency is related to increased risk of ischemic heart disease and stroke. It has been found that in men the concentration of DHEAS, as well as insulinemia, is a prognostic factor for estimating the life length $[18,19]$. Women with DHEA deficiency have lower bone mass than their peers without such deficiency. In women and men with dementia, unable to function on their own, low DHEA concentrations have been found [20]. Because DHEA deficiency is also related to lower self-esteem and depressive tendencies, attempts have been made to supplement DHEA in elderly persons with depression. A positive impact of DHEA in both sexes has been found, both in conjunction with antidepressants and in the case of monotherapy [17, 21]. Low DHEA levels have been related to a higher risk for erectile dysfunction, and a positive impact on potency in ageing men with DHEA insufficiency has also been described [22]. In several studies, an improvement of insulin sensitivity after supplementation of DHEA and a positive impact of the hormone on the fibrinolysis system have been noted. Apart from increased serum excretion and slight hirsutism in women, no significant side effects have been observed. It has to be stressed, however, that the period of the described DHEA therapies in healthy elderly people does not as a rule exceed 12-18 months. Also the number of participants in most described interventional studies was very low. Considerably longer therapies concern only patients with adrenal insufficiency, so, despite some encouraging results of studies on DHEAS common use in ageing persons, long-lasting therapy is not recommended nowadays [17].

\section{Mineralocorticoids}

In old rats, lower concentration of aldosterone in serum and lower basic aldosterone secretion from the adrenal cortex have been observed. They were caused by decreased activity of aldosterone synthase and a decreased amount of cytochrome P450scc in the cells of the glomerular layer [23]. Also in humans an age-related decrease in circulating renin and aldosterone concentrations was observed [24]. However, the evaluation of glomerular zone status in elderly people is difficult. The concentration of aldosterone in serum is determined by adrenal cortex function, cardiovascular system sufficiency, the state of the kidneys and liver, and medicine currently being taken. Works available in the literature refer mainly to the role of aldosterone in the pathogenesis of hypertension and cardiac insufficiency.

\section{Morphological changes in adrenal cortex}

Changes in concentrations of circulating corticoids are caused by the changes in morphology of the adrenals. The mass and overall thickness of the adrenal cortex decrease with age. At the same time, there occur changes in the proportions between the fascicular layer, which does not disappear, and may even slightly increase in 
volume, and the reticular layer, which becomes thinner and thinner, while its cells disperse. The clear boundary between them also disappears. In extreme cases, the cells of the zona reticularis form dispersed, weakly separated aggregations among the cells producing glucocorticoids [25]. Those changes are also expressed in the composition of enzymatic systems taking part in steroidogenesis. Activity of cytochrome P450c17 is maintained, while cytochrome $B 5$ activity decreases. As a result of those changes, the activity of 17B-hydroxylase, necessary for producing glucocorticoids, remains unaffected. At the same time, the function of 17,20-lyase (desmolase) - a trace enzyme of DHEA synthesis - decreases [26].

Apart from the changes in proportions between the layers, adrenal tumors are more frequently diagnosed. According to the analysis of autopsy research from the 1960s and 70s, in ageing adrenals small hyperplastic nodules commonly occur in the elderly. According to one of these studies, such changes were observed in $65 \%$ of autopsies [27]. In another study, single nodules of $>1 \mathrm{~cm}$ diameter were diagnosed in $8.7 \%$ of the population after 65 year of life. In the vast majority of cases, such tumors are benign, hormonally inactive lesions. With the development of imaging techniques they are more and more frequently discovered, constituting a serious clinical problem. Diagnosis of adrenal incidentaloma requires a decision concerning possible surgical treatment. If the diameter of the nodule does not exceed $4 \mathrm{~cm}$, its shape is regular, and it is homogeneous with low density in computed tomography examination, the lesion may be treated as definitively benign. However, the hormonal function of the tumor should also be assessed (evaluation of cortisol and androgen concentrations in serum, evaluation of daily excretion of free cortisol, 17-OH steroids and 17-ketosteroids, metanephrines in urine or blood, aldosterone and renin plasma activity or renin concentration in blood). The best test to exclude subclinical hypercortisolemia is a dexamethasone suppression test [29]. Subclinical form of hypercortisolemia occurs in the case of autonomous cortisol secretion from the tumor, usually at normal cortisol concentrations. No typical features of hypercortisolemia are then observed, but increased risk of developing diabetes, abdominal obesity, dyslipidemia and hypertension occurs. Elderly patients with subclinical hypercortisolemia are sometimes treated for years as patients with metabolic syndrome [30]. However, if the above-mentioned diseases are well controlled by medications, there are no absolute indications for adrenalectomy in patients over 50 [31].

\section{Conclusions}

With age, in the human adrenal cortex numerous morphological changes occur, and the production of hormones becomes different. The decrease of thick- ness of the reticular layer has been described, as well as impairment of functioning of its cells, which eventually causes the decrease of secretion of the androgens. The production of cortisol in the fascicular zone significantly increases, particularly in elderly persons with depression. An increase of the percentage of persons with nodular hyperplasia or single adrenal tumors is observed. Careful investigation of hormonal function in elderly patients, in whom both adrenal insufficiency and adrenal hyperfunction may have a subclinical form, is extremely important.

\section{Disclosure}

Author reports no conflict of interest.

\section{References}

1. Hornsby PJ. Aging of the Human Adrenal Cortex. Sci Aging Knowl Environ 2004; 35: 1-8.

2. Veldhuis JD. Changes in Pituitary Function with Aging and Implications for Patient Care. Nat Rev Endocrinol 2013; 9: 205-215.

3. Laughlin G, Barret-Connor E. Sexual Dimorphism in the Influence of Advanced Aging on Adrenal Hormone Levels: The Rancho Bernardo Study. J Clin Endocrinol Metab 2000; 85: 3561-3568.

4. Van Cauter E, Leproult R, Kupfer DJ. Effects of Gender and age on the levels and circadian rhythmicity of plasma Cortisol. J Clin Endocrinol Metab 1996; 81: 2468-2473.

5. Chahal HS, Drake WM. The endocrine system and ageing. J Pathol 2007; 211: 173-180.

6. Wilkinson CE, Petrie EC, Murray SR, et al. Human Glucocorticoid Feedback Inhibition Is Reduced in Older Individuals: Evening Study. J Clin Endocrinol Metab 2001; 86: 545-550.

7. Parker CR, Slayden SM, Azziz R, et al. Effects of Ageing in Adrenal Function in the Human: Responsiveness and Sensitivity of Adrenal Androgens and Cortisol to Adrenocorticotropin in Premenopausal and Postmenopausal Women. J Clin Endocrinol Metab 2000; 85: 48-54.

8. Van der Berghe G, Shalet SM. Critical illness as a model of hypothalamic ageing. In: Endocrine Facets of Ageing. Chadwick J, Goode J (eds.). Wiley 2002; 205-214.

9. Beale E, Zhu J, Belzberg $\mathrm{H}$. Changes in serum cortisol with age in critically ill patients. Gerontology 2002; 48: 84-92.

10. Lupien $S$, de Leon $M$, de Santi $S$, et al. Cortisol levels during human aging predict hippocampal atrophy and memory deficits. Nat Neurosci 1998; 1: 69-73.

11. Wolf O. HPA axis activity in aging women: its impact on the brain and the potential influence of estradiol. In: Hormones, Cognition and Demantia: State of Art and Emergent Therapeutic Strategies. Hogervorst E (ed.). Camridge University Press 2009; 133-140.

12. Gupta V, Lipsitz LA. Orthostatic hypotension in the elderly: diagnosis and treatment. Am J Med 2007; 120: 841-847.

13. Bornstein SR, Allolio B, Arlt W, et al. Diagnosis and Treatment of Primary Adrenal Insufficiency: An Endocrine Society Clinical Practice Guideline. J Clin Endocrinol Metab 2016; 101: 364.

14. Papierska L, Rabijewski M. Delay in diagnosis of adrenal insufficiency is a frequent cause of adrenal crisis. Int J Endocrinol 2013; 2013: 482370.

15. Hornsby PJ. Biosynthesis of DHEAS by the human adrenal cortex and its age-related decline. Ann N Y Acad Sci 1995; 774: 29-46.

16. Orentreich N. Age changes and sex differences in serum dehydroepiandrosterone sulfate concentrations throughout adulthood J Clin Endocrinol Metab 1985; 59: 551-557.

17. Samaras N, Samaras D, Frangos E, et al. A review of age-related dehydroepiandrosterone decline and its association with well-known geriatric syndromes: is treatment beneficial? Rejuvenation Res 2013; 16: 285-294.

18. Ohlsson C, Labrie F, Barrett-Connor E, et al. Low serum levels of dehydroepiandrosterone sulphate predict all-cause and cardiovascular 
mortality in elderly Swedish men. J Clin Endocrinol Metab 2010; 95 : 4406-4414.

19. Shufelt C, Bretsky P, Almeida CM, et al. DHEAS levels and cardiovascular disease mortality in postmenopausal women. J Clin Endocrinol Metab 2010; 95: 4985-4992.

20. Rudman D, Shetty KR, Mattson DE. Plasma dehydroepiandrosterone sulfate in nursing home men. J Ann Geriatr Soc 1990; 38: 421-427.

21. Schidt PJ, Daly RC, Bloch M, et al. Dehydroepiandrosterone Monotherapy in Midlife-Onset Major and Minor Depression. Arch Gen Psychiatry 2005; 62: 154-162.

22. Reiter W, Pycha A, Schatzl G, et al. Dehydroepiandrosterone in the treatment of erectile dysfunction: a prospective, double-blind, randomized, placebo-controlled study. Urology 1999; 53: 590-595.

23. Kau MM, Chen JJ, Wang SW, et al. Age-related impairment of aldosterone secretion in zona glomerulosa cells of ovariectomized rats. J Investig Med 1999; 47: 425-432.

24. Weidmann P, de Myttenaere-Bursztein S, Maxwell MH, de Lima J. Effect on aging on plasma renin and aldosterone in normal man. Kidney Int 1975; 8: 325-333.

25. Parker CR. Aging alters zonation in the adrenal cortex of men. J Clin Endocrinol Metab 1997; 82: 3898-3901.

26. Dharia S. Effects of Ageing on Cytochrome B5 Expresssion in the Human adrenal Gland. J Clin Endocrinol Metab 2005; 90: 4357-4361.

27. Dobble JW. Adrenocortical nodular hyperplasia: The aging adrenal. J Pathol 1969; 99: 1-18.

28. Hedeland $\mathrm{H}$. On the prevalence of Adrenocortical adenomas in an autopsy material in relation to hypertension and diabetes. Acta Med Scand 1968; 211-214.

29. Bednarczuk T, Bolanowski M, Sworczak K. Adrenal incidentaloma in adults - management recommendations by the Polish Society of Endocrinology. Endokrynol Pol 2016; 67: 234-258.

30. Terzolo M, Pia A, Alì A, et al. Adrenal incidentaloma: a new cause of the metabolic syndrome? J Clin Endocrinol Metab 2002; 87: 998-1003.

31. Fassnacht M, Arlt W, Bancos I, et al. Management of adrenal incidentalomas: European Society of Endocrinology Clinical Practice Guideline in collaboration with the European Network for the Study of Adrenal Tumors. Eur J Endocrinol 2016; 175: G1-34. 